\title{
Preoperative Venous Thromboembolism in Orthopedic Oncology Patients: Incidence, Risk Factors, and D-Dimer Cutoff Value
}

\author{
Kenta Hayashida \\ Yokohama City University \\ Yokohama City University \\ Keiju Saito \\ Yokohama City University \\ Shintaro Fujita \\ Yokohama City University \\ Hyonmin Choe \\ Yokohama City University \\ Masanobu Takeyama \\ Yokohama City University \\ Yutaka Inaba \\ Yokohama City University
}

Yusuke Kawabata ( $\square$ yusuke0807kawabata@yahoo.co.jp )

\section{Research Article}

Keywords: preoperative venous thromboembolism, VTE, deep vein thrombosis, DVT, malignant musculoskeletal tumor, orthopedic oncology, D-dimer

Posted Date: September 20th, 2021

DOI: https://doi.org/10.21203/rs.3.rs-863648/v1

License: (c) (1) This work is licensed under a Creative Commons Attribution 4.0 International License. Read Full License 


\section{Abstract}

Background: Venous thromboembolism (VTE) is known as one of the major complications in patients with malignant tumors and orthopedic disorders. Patients undergoing surgery for orthopedic oncology are at an increased risk for thromboembolic events. However, there are limited studies regarding the incidence and risk factors for preoperative VTE in orthopedic oncology patients.

Methods: The aim is to determine the incidence and risk factors of preoperative VTE by screening with Ddimer. We retrospectively reviewed the medical records of 270 patients who underwent surgical procedures, including biopsy for orthopedic oncology, had preoperative D-dimer levels measured and were subsequently screened for VTE by lower extremity venous ultrasonography and contrast-enhanced computed tomography (CT) scans if necessary. Statistical analyses were performed to examine the incidence and risk factors for VTE. Receiver operating characteristic (ROC) analysis was performed to verify the cutoff value of D-dimer for the diagnosis of VTE.

Results: A total of 199 patients (103 with primary soft tissue sarcomas, 38 with primary bone sarcomas, 46 with metastatic tumors and 12 with hematologic malignancies) were included. The value of D-dimer was high in 79 patients. VTE was detected in 19 patients (9.5\%). Multivariate analysis indicated that, age $\geq 60$ years $(P=0.021)$, and tumor location in the lower limbs $(P=0.048)$ were independent risk factors for VTE. ROC analysis showed that the cutoff value of D-dimer for the diagnosis of VTE was $1.53 \mu \mathrm{g} / \mathrm{mL}$ (sensitivity of $89.5 \%$ and specificity of $79.4 \%$ ).

Conclusions: Our study indicated that age and tumor location in the lower limbs were independent risk factors for preoperative VTE in orthopedic oncology patients. D-dimer was not significantly different in the multivariate analysis because D-dimer is affected by a wide variety of conditions, such as malignancy and aging. Patients with high D-dimer levels and the abovementioned risk factors are at increased risk of preoperative VTE, and additional ultrasonography should be considered.

Trial registration: Our study was approved by the institutional review board. The registration number is B200600056. The registration date was July 13, 2020.

\section{Background}

Venous thromboembolism (VTE), including deep vein thrombosis (DVT) and pulmonary embolism (PE), is a major complication in orthopedic surgery. VTE is also known to be associated with malignancy, which increases the risk of VTE by 2- to 7-fold [1-3]. Several mechanisms may be involved in the promotion of thromboembolic events in cancer patients [4]. Because the risk of VTE is reported to be particularly high in the first few months after the diagnosis of malignancy [3], it is important to assess VTE in patients with malignancy before starting treatment.

D-dimer, the breakdown product of stabilized fibrin, is frequently used to screen for VTE because there is well-established evidence that a D-dimer value below $0.5 \mu \mathrm{g} / \mathrm{mL}$ has an exceedingly high negative 
predictive value for the exclusion of PE [5-7]. The combination of clinical symptoms, D-dimer, and contrast-enhanced computed tomography (CT) has made it possible to diagnose $97.9 \%$ of VTE cases [8, 9].

The incidence of perioperative VTE in orthopedic oncology patients has been reported to range from $2.7 \%$ to $22 \%$ [10-18]. Various factors, such as age, size and location of the tumor, chemotherapy, Ewing sarcoma family, metastasis, pathological fracture, and type of surgery, are suggested to be related to the occurrence of VTE [10-14,17-20]. However, most previous studies analyzed postoperative patients who underwent orthopedic surgery $[10,12-17,19]$. In addition, routine screening for VTE was not considered, so the reported rate of VTE represented only those that were clinically symptomatic $[12,14]$.

Because such limitations may cause an underestimation of the incidence of VTE, only limited evidence is available for understanding preoperative VTE in orthopedic oncology patients. Therefore, we investigated the incidence and risk factors for VTE in these patients and considered that the best screening method for VTE was the combination of D-dimer and imaging examination.

\section{Methods}

After approval by our institutional review board, we retrospectively reviewed the medical records of patients in our institution between January 2014 and June 2020. A total of 199 of 270 orthopedic oncology patients who underwent orthopedic surgical procedures, including open biopsy, and were assessed for preoperative VTE according to a flowchart prescribed by our institution were included (Figure 1). VTE was screened by measuring preoperative D-dimer levels and subsequently performing lower extremity venous ultrasonography and/or contrast-enhanced CT scans. D-dimer levels were measured using a latex agglutination turbidimetric immunoassay. Owing to the sensitivity of this assay, levels $<0.50 \mu \mathrm{g} / \mathrm{mL}$ were considered $0.50 \mu \mathrm{g} / \mathrm{mL}$. Ultrasonography and CT scans were performed if the D-dimer level was $\geq 1.0 \mu \mathrm{g} / \mathrm{mL}$. In this study, DVT in the lower extremities that involved the popliteal vein and above was defined as the proximal type; DVT involving the area below the popliteal vein was defined as the distal type. We excluded patients with a benign condition, with a past history of VTE, who were treated with direct oral anticoagulants (DOACs) or heparin before the assessment of VTE, with reoperation within 3 months, and without the measurement of preoperative D-dimer levels. If the D-dimer level was $\geq 1.0 \mu \mathrm{g} / \mathrm{mL}$, patients without lower extremity venous ultrasonography or contrast-enhanced CT examinations were also excluded.

The clinical profiles of VTE patients were assessed retrospectively. The anatomical location of the tumor was defined as the lower limbs and others. The lower limbs included the pelvis, hip, thigh, knee, lower leg, ankle, and foot. The tumor location was also assessed by the depth of the tumor. A soft tissue tumor located on the surface of the fascia was defined as superficial. Soft tissue tumors deeper than the fascia or bone tumors were defined as deep. The patients comprised 103 males and 77 females, with a mean age of 60.9 years (range 14-91 years). The tumor location was the lower limbs in 133 patients. The tumors comprised 103 primary soft tissue sarcomas, 38 primary bone sarcomas, 46 metastatic tumors 
and 12 hematologic malignancies. The pathological diagnoses of the patients are summarized in Table 1.

The Mann-Whitney test was used for continuous variables, and the chi-squared test or Fisher's exact test was used for categorical variables. Logistic regression analysis was used as a multivariate analysis. Receiver operating characteristic (ROC) analysis was performed to verify the cutoff value of D-dimer for the diagnosis of VTE. A p-value of less than 0.05 was considered significant. All statistical analyses were performed using R (R Core Team (2020); R: A language and environment for statistical computing. R Foundation for Statistical Computing, Vienna, Austria).

\section{Results}

The value of D-dimer was $\geq 1.0 \mu \mathrm{g} / \mathrm{mL}$ in $79(39.7 \%)$ patients. The overall incidence of preoperative VTE detected by ultrasonography and contrast-enhanced CT scans was $9.5 \%$ (19 patients) (Table 2). VTE was detected in $8.7 \%$ and $7.9 \%$ of patients with soft tissue and bone sarcomas, respectively. The incidence of VTE in patients with metastasis was $10.9 \%$. Proximal VTE and PE were detected in $3(2.5 \%)$ and $2(1.7 \%)$ patients, respectively. The clinical profiles of the patients with VTE are summarized in Table 3 . The patients comprised 10 males and 9 females, with a mean age of 59.7 years (range 19-91). The tumor was located on the lower limbs in 17 patients, accounting for $89.5 \%$ of the patients with VTE. The mean D-dimer level was 2.64 (range 1.05-11.72) $\mu \mathrm{g} / \mathrm{mL}$. Among the patients with VTE, 9 patients had soft tissue sarcomas, and 3 patients had bone sarcomas. The cases of soft tissue sarcoma included 4 myxofibrosarcoma, 3 undifferentiated pleomorphic sarcoma (UPS), and 2 leiomyosarcoma. The tumor was located on the surface of the fascia in 4 patients and deeper than the fascia in 5 patients. The cases of bone sarcoma included 2 UPS of the bone and 1 osteosarcoma. Seven patients had bone metastasis and hematologic malignancy. ROC analysis showed that the cutoff value of D-dimer for the diagnosis of VTE was $1.53 \mu \mathrm{g} / \mathrm{mL}$. The area under the curve was 0.86 . The sensitivity and specificity were $89.5 \%$ and $79.4 \%$, respectively.

The risk factors for VTE were analyzed (Table 4). In the univariate analysis, age $(P=0.0039)$, age $\geq 60$ years $(P=0.011)$, tumor location in the lower limbs $(P=0.038)$, and $D$-dimer $(P<0.001)$ were related to the incidence of VTE. Sex, age, body mass index, size, origin, performance status, pathological fracture, and hematologic status, including hemoglobin, white blood cells, platelets, and C-reactive protein, were not significant risk factors. The multivariate analysis indicated that age $(P=0.012)$, age $\geq 60$ years $(P=$ 0.021), and tumor location in the lower limbs $(P=0.048)$ were independent risk factors for VTE. D-dimer was not significant in the multivariate analysis $(P=0.51)$.

\section{Discussion}

We investigated the incidence of preoperative VTE in orthopedic oncology patients. The most important finding in this study was that more orthopedic oncology patients could potentially have VTE than reported by previous studies. Nineteen of 199 patients (9.5\%) had VTE detected in this study. The 
incidence was higher than the rate of perioperative VTE reported in previous studies [10-15]. Several conditions that differed from those in previous studies may have influenced the results. First, routine screening for VTE was examined in this study. We performed DVT assessment in combination with routine D-dimer measurement, lower extremity ultrasonography and contrast-enhanced CT scans. This screening detected patients with asymptomatic VTE who were not diagnosed by physical symptoms. The necessity of anticoagulation for those patients is controversial, but Galanaud et al. reported that patients with cancer-related isolated distal DVT have a prognosis that is similar to that of patients with cancerrelated isolated proximal DVT and a dramatically poorer prognosis than those with isolated distal DVT without cancer [21]. Therefore, it is important for the management of preoperative orthopedic oncology patients to recognize that more patients have asymptomatic VTE. Second, our study included patients with metastasis and hematologic malignancy. The rate of DVT in surgical oncology patients receiving no prophylaxis was reported to be $35.2 \%$ [22]. The cancer type affects the incidence of VTE in adolescent and young adult patients. Therefore, oncology patients, except for sarcoma patients, might have a different total incidence of VTE.

Several risk factors associated with an increased risk of preoperative VTE in orthopedic oncology patients have been found. Our study showed that increasing age and tumor location are independent risk factors. Age has been suggested to be associated with an increased risk of VTE [10,17]. Kim et al. reported an odds ratio of 5.84 in patients older than 60 years [10]. Our results showed a similar trend, and only 2 patients with VTE were younger than 60 years. Patients with bone or soft tissue sarcomas located in the hip or thigh have been suggested to have an increased risk of VTE [14]. Yamaguchi et al.

demonstrated a high incidence of VTE $(22 \%)$ in patients after resection of musculoskeletal tumors of the lower limb [17]. Since surgery involving the pelvis has been associated with the development of proximal DVT [19], patients with tumors in the lower limbs are suggested to be increased at risk of VTE occurrence. In this study, 17 of 19 VTE patients had tumors in the lower limbs (89.5\%), and the two VTE patients with tumors in the upper extremities were both older than 90 years.

D-dimer showed an association with preoperative VTE in univariate analysis. However, no significant difference was observed in multivariate analysis due to the influence of increased age on the D-dimer level. D-dimer needs to be corrected by age, and the value of D-dimer itself should be evaluated carefully $[5,7]$. In addition, the D-dimer cutoff value in cancer patients was reported to be higher than that commonly used [23]. The cutoff value of D-dimer for the diagnosis of VTE was determined to be $1.53 \mu \mathrm{g} / \mathrm{mL}$. This value was almost the same as that used in other cancer types [24,25]. The appropriate consideration of D-dimer limits the overuse and added cost of ultrasonography without a negative impact [26]. If D-dimer is in excess of $1.53 \mu \mathrm{g} / \mathrm{mL}$, especially in patients with the risk factors that were suggested in this study, such as older age and tumor location in the lower limbs, additional assessment of VTE by lower extremity ultrasonography and contrast-enhanced CT should be considered.

Our study has several limitations. First, our study was retrospective and had a relatively small sample size. Some predictive variables may not have had sufficient statistical power, and some important variables may have been ignored. Second, patients with metastasis and hematologic malignancy were 
included. Regarding the evaluation of VTE risk in bone and soft tissue sarcoma patients, these malignancies should be evaluated in separate categories with sufficient sample sizes. Third, lower extremity ultrasonography and contrast-enhanced CT for VTE evaluation were not unified.

\section{Conclusions}

Our study indicated that age and tumor location in the lower limbs were independent risk factors for preoperative VTE in orthopedic oncology patients. D-dimer was not significantly different in the multivariate analysis because D-dimer is affected by a wide variety of conditions, such as malignancy and aging. However, D-dimer was useful for exclusion diagnosis due to its high sensitivity. Patients with high D-dimer levels and the abovementioned risk factors are at increased risk of preoperative VTE and should be considered for additional assessment.

\section{List Of Abbreviations}

\begin{tabular}{|ll|}
\hline Abbreviations & Full spelling \\
\hline CT & computed tomography \\
\hline DOAC & direct oral anticoagulant \\
\hline DVT & deep vein thrombosis \\
\hline PE & pulmonary embolism \\
\hline ROC & receiver operating characteristic \\
\hline VTE & venous thromboembolism \\
\hline
\end{tabular}

\section{Declarations}

Ethics approval and consent to participate: This research has been approved by the IRB of the Yokohama City University Hospital. The approval number is B200600056. All methods were carried out in accordance with relevant guidelines and regulations. Informed consent was obtained from all participants or, if participants are under 16, from a parent and/or legal guardian.

Consent for publication: Consent for publication was obtained from all participants or, if participants are under 16, from a parent and/or legal guardian.

Availability of data and materials: Data sharing is not applicable to this article as no datasets were generated or analysed during the current study.

Competing interests: The authors declare that they have no competing interests.

Funding: The authors declare that they have no funding. 
Authors' contributions: Kenta Hayashida M.D.: design of the work and have drafted the work, Yusuke Kawabata M.D. Ph.D.: substantial contributions to the conception, Keiju Saito M.D.: the acquisition, analysis, Shintaro Fujita M.D.: interpretation of data, Hyonmin Choe M.D., Ph.D.: substantial contributions to the conception, Masanobu Takeyama M.D. Ph.D.: substantial contributions to the conception, Yutaka Inaba M.D. Ph.D.: have approved the submitted version.

Acknowledgements: Not applicable

\section{References}

1. Stein PD, Beemath A, Meyers FA, Skaf E, Sanchez J, Olson RE. Incidence of venous thromboembolism in patients hospitalized with cancer. Am J Med. 2006;119(1):60-8.

2. Heit JA, Silverstein MD, Mohr DN, Petterson TM, O'Fallon WM, Melton 3rd LJ. Risk factors for deep vein thrombosis and pulmonary embolism: a population-based case-control study. Arch Intern Med. 2000;160(6):809-15.

3. Blom JW, Doggen CJM, Osanto S, Rosendaal FR. Malignancies, Prothrombotic Mutations, and the Risk of Venous Thrombosis. JAMA. 2005;293(6):715-22.

4. Donnellan E, Khorana AA. ï» ¿Cancer and Venous Thromboembolic Disease: A Review. Oncologist. 2017;22(2):199-207.

5. Righini M, Perrier A, Moerloose PD, Bounameaux H. D-Dimer for venous thromboembolism diagnosis: 20 years later. J Thromb Haemost. 2008;6(7):1059-71.

6. Nisio MD, Squizzato A, Rutjes AWS, Büller HR, Zwinderman, Bossuyt PMM. Diagnostic accuracy of Ddimer test for exclusion of venous thromboembolism: a systematic review. J Thromb Haemost. 2007;5(2):296-304.

7. Nybo M, Hvas AM. Age-adjusted D-dimer cut-off in the diagnostic strategy for deep vein thrombosis: a systematic review. Scand J Clin Lab Invest. 2017;77(8):568-573.

8. Wells PS, Anderson DR, Rodger M, Ginsberg JS, Kearon C, Gent M, Turpie AG, Bormanis J, Weitz J, Chamberlain M, Bowie D, Barnes D, Hirsh J. ï»¿Derivation of a simple clinical model to categorize patients' probability of pulmonary embolism: increasing the model's utility with the SimpliRED Ddimer. Thromb Haemost. 2000;83(3):416-20.

9. van Belle A, Büller HR, Huisman MV, Huisman PM, Kaasjager K, Kamphuisen PW, Kramer MHH, Kruip MJHA, J Erp JMK, Leebeek FWG, Nijkeuter M, Prins MH, Sohne M, Tick LW, Christopher Study Investigators. Effectiveness of managing suspected pulmonary embolism using an algorithm combining clinical probability, D-dimer testing, and computed tomography. JAMA. 2006;295(2):1729.

10. Kim SM, Park JM, Shin SH, Seo SW. Risk factors for post-operative venous thromboembolism in patients with a malignancy of the lower limb. Bone Joint J. 2013 95-B(4):558-62.

11. Morii T, Mochizuki K, Tajima T, Aoyagi T, Satomi K. Venous thromboembolism in the management of patients with musculoskeletal tumor. J Orthop Sci. 2010;15(6):810-5. 
12. Damron TA, Wardak Z, Glodny B, Grant W. Risk of venous thromboembolism in bone and soft-tissue sarcoma patients undergoing surgical intervention: a report from prior to the initiation of SCIP measures. J Surg Oncol. 2011 Jun 1;103(7):643-7.

13. Kaiser CL, Freehan MK, Driscoll DA, Schwab JH, Bernstein KDA, Lozano-Calderon SA. Predictors of venous thromboembolism in patients with primary sarcoma of bone. Surg Oncol. 2017;26(4):506510.

14. Mitchell SY, Lingard EA, Kesteven P, McCaskie AW, Gerrand $\mathrm{CH}$. Venous thromboembolism in patients with primary bone or soft-tissue sarcomas. J Bone Joint Surg Am. 2007;89(11):2433-9.

15. Singh VA, Yong LM, Vijayananthan A. Is DVT prophylaxis necessary after oncology lower limb surgery? A pilot study. Springerplus. 2016;5(1):943.

16. Tuy B, Bhate $C$, Beebe $K$, Patterson F, Benevenia J. IVC filters may prevent fatal pulmonary embolism in musculoskeletal tumor surgery. Clin Orthop Relat Res. 2009;467(1):239-45.

17. Yamaguchi T, Matsumine A, Niimi R, Nakamura T, Asanuma K, Hasegawa M, Sudo A. Deep-vein thrombosis after resection of musculoskeletal tumours of the lower limb. Bone Joint J. 2013;95$\mathrm{B}(9): 1280-4$.

18. Li M, Guo Q, Hu W. Incidence, risk factors, and outcomes of venous thromboembolism after oncologic surgery: A systematic review and meta-analysis. Thromb Res. 2019;173:48-56.

19. Nathan SS, Simmons KA, Lin PP, Hann LE, Morris CD, Athanasian EA, Boland PJ, Healey JH. Proximal deep vein thrombosis after hip replacement for oncologic indications. J Bone Joint Surg Am. 2006;88(5):1066-70.

20. Ogura K, Yasunaga H, Horiguchi H, Ohe K, Kawano H. Incidence and risk factors for pulmonary embolism after primary musculoskeletal tumor surgery. Clin Orthop Relat Res. 2013;471(10):3310-6.

21. Galanaud JP, Sevestre MA, Pernod G, Genty C, Richelet S, Kahn SR, Boulon C, Terrisse H, Quéré I, Bosson JL. Long-term outcomes of cancer-related isolated distal deep vein thrombosis: the OPTIMEV study. J Thromb Haemost. 2017;15(5):907-916.

22. Leonardi MJ, McGory ML, Ko CY. A systematic review of deep venous thrombosis prophylaxis in cancer patients: implications for improving quality. Ann Surg Oncol. 2007;14(2):929-36.

23. Chen C, Li G, Liu YD, Gu YJ. A new D-dimer cutoff value to improve the exclusion of deep vein thrombosis in cancer patients. Asian Pac J Cancer Prev. 2014;15(4):1655-8.

24. Kawaguchi R, Furukawa N, Kobayashi H. Cut-off value of D-dimer for prediction of deep venous thrombosis before treatment in ovarian cancer. J Gynecol Oncol. 2012;23(2):98-102.

25. Osaki T, Saito H, Fukumoto Y, Kono Y, Murakami Y, Shishido Y, Kuroda H, Matsunaga T, Sato K, Hirooka Y, Fujiwara Y. Risk and incidence of perioperative deep vein thrombosis in patients undergoing gastric cancer surgery. Surg Today. 2018;48(5):525-533.

26. Mousa AY, Broce M, Gill G, Kali M, Yacoub M, AbuRahma AF. Appropriate use of D-dimer testing can minimize over-utilization of venous duplex ultrasound in a contemporary high-volume hospital. Ann Vasc Surg. 2015;29(2):311-7. 


\section{Tables}

Due to technical limitations, table 1,2,3,4 is only available as a download in the Supplemental Files section.

\section{Figures}

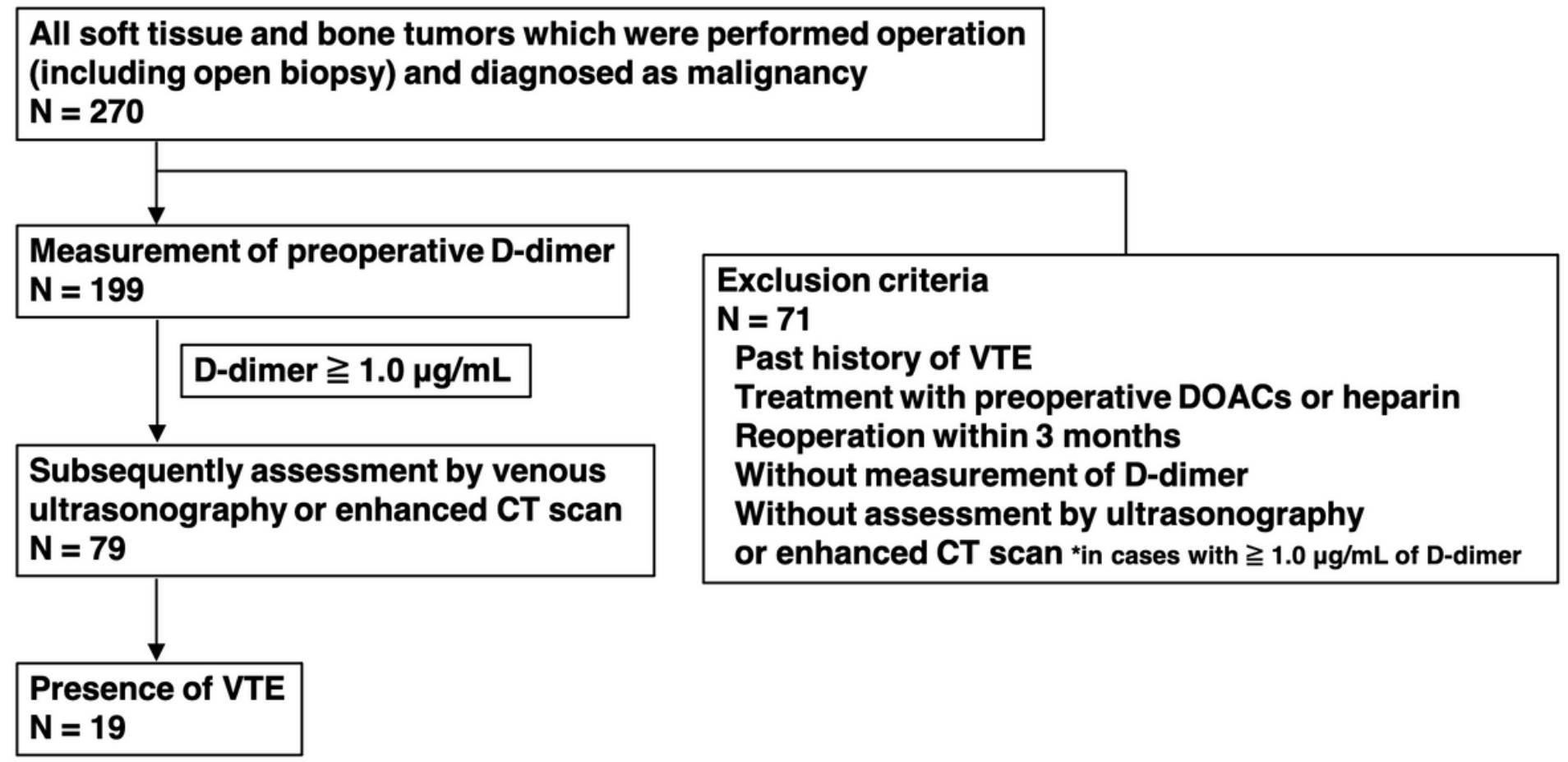

Figure 1

Flow chart of the assessment for VTE in this study Patients were evaluated using D-dimer screening, lower extremity venous ultrasonography, and contrast-enhanced CT scan. A total of 71 patients were excluded according to exclusion criteria.

\section{Supplementary Files}

This is a list of supplementary files associated with this preprint. Click to download.

- Table1.xlsx

- Table2.xlsx

- Table3.xlsx

- Table4.xIsx 